\title{
Stationary Interference Cancellation in Upstream Coordinated DSL Using a Turbo-MMSE Receiver
}

\author{
Issam Wahibi, ${ }^{1,2}$ Meryem Ouzzif, ${ }^{1}$ Jérôme Le Masson, ${ }^{1}$ and Samir Saoudi ${ }^{2}$ \\ ${ }^{1}$ Orange Laboratories, 2 avenue Pierre Marzin, 22307 Lannion, France \\ ${ }^{2}$ Département Signal et Communications, INSTITUT Telecom, TELECOM Bretagne, Technopôle Brest-Iroise, \\ CS 83818, 29238 Brest Cedex 3, France
}

Correspondence should be addressed to Meryem Ouzzif, meryem.ouzzif@francetelecom.com

Received 1 April 2008; Accepted 19 July 2008

Recommended by Marina Mondin

\begin{abstract}
We investigate the scenario of an upstream coordinated DSL transmission in presence of spatial-correlated noise. Joint signal processing helps mitigate this noise and reduce internal interference effects between coordinated users. We propose to couple noise whitening with a mean-squared error iterative receiver in order to approach the matched filter bound of the DSL coordinated system. The convergence of the iterative scheme in this scenario is predicted using EXIT charts under realistic transmission conditions.
\end{abstract}

Copyright (c) 2008 Issam Wahibi et al. This is an open access article distributed under the Creative Commons Attribution License, which permits unrestricted use, distribution, and reproduction in any medium, provided the original work is properly cited.

\section{INTRODUCTION}

Faced with the mounting need of residential and professional customers to benefit from new high data-rate multimedia services, access network operators are improving their offers thanks to diverse high-speed technologies. Very high speed digital subscriber line (VDSL2) allows tens of megabits per second to be sent to customers over copper lines for short distances. This technique, associated with an optical termination situated close to the customer premises, can supply this need while saving on infrastructure costs. In this configuration, far-end crosstalk (FEXT) coupling between copper lines can represent a major performance limitation.

Crosstalk, in the case of a coordinated DSL transmission, can be separated into two categories. The first, called indomain crosstalk, comes from the vectored system whereas the second category of crosstalk, called out-of-domain crosstalk, originates from outside the coordinated system when two or more access network operators share the same binder [1]. When the DSL systems transmit continuously, the crosstalk noise can be considered stationary. Several techniques have been proposed for in-domain crosstalk cancellation in the upstream link. In the literature, Ginis and Cioffi use a zero forcing-successive interference cancellation (ZF-SIC) structure for FEXT cancellation [2], while Cen- drillon et al. show that a linear zero-forcing (ZF) canceller achieves near optimal performance [3].

Out-of-domain crosstalk or equivalently external interference presents a spatial correlation on the receiver side. A whitening filter based on the Cholesky decomposition is applied to mitigate its impact and is followed by a successive interference cancellation (SIC) structure to help reduce the inherent interference of the equivalent channel [1]. A power allocation method that mitigates the external crosstalk effect in vectored DSL systems has been proposed in [4].

In the presence of strong external interference, the previously presented interference vectored cancellation schemes perform far from the optimum. In this paper, we propose to carry out after the whitening filter an iterative processing based on the minimum mean-squared error (MMSE) criterion using a priori information [5-7]. The iterative detection of multiple-input multiple-output (MIMO) signals using the MMSE detector is presented in [8].

The remainder of the paper is organized as follows. The system model is described in Section 2. Maximum throughput upper bound for coordinated DSL systems in presence of external interference is derived in Section 3. The ZF-SIC and the linear iterative MMSE detectors are examined in Section 4. Numerical results are analyzed in Section 5 and conclusions are drawn in Section 6. 


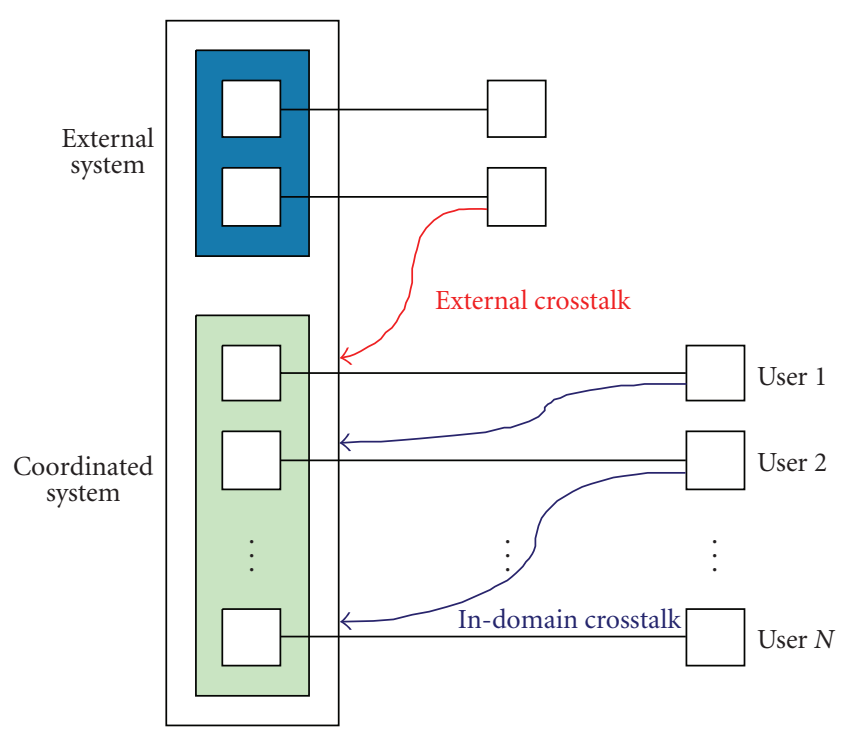

FIgURe 1: External crosstalk environment.

\section{SYSTEM MODEL}

A coordinated DSL system is depicted in Figure 1. N coordinated lines are colocated in the same Optical Network Unit (ONU). Because of crosstalk between pairs of the same binder, the coordinated signals interfere with each other and undergo interference from $L$ different external sources. The $N$ coordinated lines can benefit from a joint signal processing at the receiver side in the upstream direction.

Such as the recently adopted standard VDSL2, the transmission is based on a discrete multitone (DMT) modulation applied to data that were first coded and interleaved before being mapped to complex QAM symbols. According to the known DMT modulation principle, the constellation size is determined on each tone as a function of its signalto-noise ratio. It is assumed that the cyclic prefix length exceeds the maximum delay of the channel and that the coordinated transmissions are perfectly synchronized. Thus, the transmission can be modelled in the frequency domain. For each tone $k$, the received signal $\mathbf{y}^{k}$ is therefore written as

$$
\mathbf{y}^{k}=\mathbf{H}^{k} \mathbf{x}^{k}+\mathbf{A}^{k} \mathbf{z}^{k}+\mathbf{w}^{k}, \quad k=1, \ldots, K,
$$

where $K$ is the number of tones. The vector $\mathbf{x}^{k}=$ $\left[x_{1}^{k}, \ldots, x_{N}^{k}\right]^{T}$ contains the complex symbols transmitted on tone $k$ for the $N$ coordinated users. The vector $\mathbf{z}^{k}=$ $\left[z_{1}^{k}, \ldots, z_{L}^{k}\right]^{T}$ contains the frequency components of the external transmitted signals on tone $k . \mathbf{w}^{k}$ is the vector of additive white Gaussian noise (AWGN) elements on each line. If the transmit power spectral density (PSD) on each coordinated line $i$ on tone $k$ is denoted by $\sigma_{x_{i}^{k}}^{2}$, the autocorrelation of the transmitted spatially uncorrelated signal is a diagonal matrix $\mathbf{D}_{\mathbf{x}^{k}}=\mathscr{E}\left\{\mathbf{x}^{k} \mathbf{x}^{k^{H}}\right\}=\operatorname{diag}\left(\sigma_{x_{1}^{k}}^{2}, \ldots, \sigma_{x_{N}^{k}}^{2}\right)$. In a similar way, the external sources are spatially uncorrelated and their autocorrelation matrix is $\mathbf{D}_{\mathbf{z}^{k}}=\mathscr{E}\left\{\mathbf{z}^{k^{k}} \mathbf{z}^{H}\right\}=$ $\operatorname{diag}\left(\sigma_{z_{1}^{k}}^{2}, \ldots, \sigma_{z_{L}^{k}}^{2}\right)$. The white noise PSD is assumed equal for the different coordinated lines. Therefore, the noise autocorrelation matrix is $\mathscr{E}\left\{\mathbf{w}^{k} \mathbf{w}^{k^{H}}\right\}=\sigma_{\mathbf{w}^{k}}^{2} \mathbf{I}_{N}$. The matrix $\mathbf{H}^{k}$ is the frequency response of the coordinated MIMO channel on tone $k$. The diagonal element $h_{i, i}^{k}$ of matrix $\mathbf{H}^{k}$ is the attenuation coefficient of the line $i$ and the off-diagonal element $h_{i, j}^{k}$ is the FEXT coupling coefficient between transmitter $j$ and receiver $i$ on tone $k$. Thanks to the physical properties of the cable, $\mathbf{H}^{k}$ is diagonally dominant. This property is inherent to the fact that the direct channel is stronger than the crosstalk coupling channels which implies diagonal elements greater than the off-diagonal elements in the channel matrix. The $N \times L$ matrix $\mathbf{A}^{k}$ contains the coupling coefficients between the external sources and the coordinated lines in the frequency domain. For reasons of clarity, the tone index is dropped in the following sections.

\section{NOISE MITIGATION AND MAXIMUM THROUGHPUT UPPER BOUND}

The resulting noise term $\mathbf{n}=\mathbf{A z}+\mathbf{w}$ in (1) is spatially correlated. Its different components cannot be directly known by the receiver but its covariance matrix $R_{\mathrm{nn}}$ can be measured. It is written as follows:

$$
R_{\mathbf{n n}}=E\left\{\mathbf{n n}^{H}\right\}=\mathbf{A D}_{\mathbf{z}} \mathbf{A}^{H}+\sigma_{\mathbf{w}}^{2} \mathbf{I}_{\mathbf{N}} .
$$

In this section, an upper bound of the multiuser DSL system maximum throughput is derived when noise whitening is carried out.

\subsection{Noise whitening}

Noise mitigation is obtained through linear filtering of the received signal in the frequency domain. The noise part of the resulting whitened signal has a diagonal correlation matrix. The computation of the whitening filter can be carried out using different methods among which two are described. The first method is based on the Cholesky decomposition of the noise correlation matrix $R_{\mathrm{nn}}$. The whitening filter can be expressed by

$$
\mathbf{W}=\mathbf{G}^{-1} \text {, }
$$

where $R_{\mathbf{n n}}=\mathbf{G G}^{H}$ and $\mathbf{G}$ is a lower triangular matrix. The upper triangular structure of the whitening filter allows successive noise cancelling to be carried out when the channel matrix $\mathbf{H}$ is diagonal as proposed in [1].

The second method is based on the inverse square root of the noise covariance matrix $R_{\mathrm{nn}}$ given by

$$
\mathbf{W}=\left(R_{\mathrm{nn}}\right)^{-1 / 2} \text {. }
$$

Eldar and Oppenheim show in [9] that this solution minimizes the mean-squared error between the original and whitened data. Therefore, it is used as a whitening solution with the iterative receiver described in the next section. In order to avoid matrix inversion, the computation of the noise whitening filter could be implemented iteratively as suggested in [10]. The received signal after whitening is expressed by

$$
\check{\mathbf{y}}=\mathbf{W y}=\check{\mathbf{H}} \mathbf{x}+\check{\mathbf{n}} .
$$




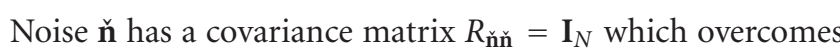
the noise correlation due to external noise in DSL coordinated systems. Unfortunately, the equivalent channel matrix H looses the diagonal dominance property useful for linear crosstalk cancellation.

\subsection{Matched filter bound (MFB)}

In order to determine the matched filter bound of the multiuser coordinated DSL receiver, a single user is assumed to be transmitting. At the receiver side, the signal is received on the direct line and on the other lines belonging to the coordinated system through crosstalk between lines. Thanks to whitening at the reception, the noise is uncorrelated and has the same power level on the different lines. This system is therefore equivalent to a channel with $N$ diversity branches. It is shown in [11] that the maximum ratio combiner (MRC) is the optimum receiver for such a transmission scheme. The MRC linearly combines the individually received branch signals so as to maximize the signal-to-noise ratio for the considered user. The MRC output signal for user $i$ can be written as

$$
y_{i}^{\mathrm{MFB}}=\sum_{j=1}^{N}\left|\check{h}_{j, i}\right|^{2} x_{i}+\sum_{j=1}^{N} \check{h}_{j, i}^{*} \check{n}_{j}
$$

With the assumption that the different users of the coordinated system do not interfere with each other, the maximum throughput of user $i$ on a given tone is expressed as

$$
C_{i}^{\mathrm{MFB}}=\log _{2}\left(1+\frac{1}{\Gamma} \sum_{j=1}^{N}\left|\check{h}_{j, i}\right|^{2} \sigma_{x_{i}}^{2}\right),
$$

where $\Gamma$ is the is the signal-to-noise ratio gap to capacity.

In practice, all the coordinated users transmit simultaneously and interfere with each other. These interferences have to be dealt with using signal processing techniques that can improve the system performance upper bounded by the maximum throughput (7).

\section{MULTIUSER DETECTION}

After whitening as described in Section 3, the different transmitted signals have to be estimated from the whitened signal y̌ expressed by (5). In such a scenario, the linear processing for the coordinated upstream DSL system proposed in [3] is no longer efficient since the equivalent channel matrix $\check{\mathbf{H}}$ is not diagonally dominant. In this section, we present two multiuser detection methods that will be investigated in this transmission configuration. The first method uses successive interference cancellation based on ZF criterion as suggested in [2] for vectored DSL systems. The second method consists of an iterative MMSE receiver as proposed for wireless MIMO systems in [8].

\subsection{ZF-based SIC}

The successive interference canceller investigated for the multiuser detection is based on the QR decomposition of the equivalent channel matrix after whitening as follows:

$$
\check{\mathbf{H}}=\mathbf{Q R},
$$

where $\mathbf{Q}$ is a unitary matrix and $\mathbf{R}$ is upper triangular. The whitened vector $\check{\text { y }}$ is multiplied by matrix $\mathbf{Q}^{H}$ which results in the following output:

$$
\overline{\mathbf{y}}=\mathbf{Q}^{H} \check{\mathbf{y}}=\mathbf{R} \mathbf{x}+\overline{\mathbf{n}},
$$

where $\overline{\mathbf{n}}=\mathbf{Q}^{H} \check{\mathbf{n}}$ and $R_{\overline{\mathbf{n}} \overline{\mathbf{n}}}=\mathbf{I}_{N}$. Since matrix $\mathbf{R}$ is upper triangular, successive interference cancellation can be carried out as follows:

$$
\hat{x}_{i}=\frac{1}{r_{i, i}}\left(\bar{y}_{i}-\sum_{j=i+1}^{N} r_{i, j} \operatorname{dec}\left(\hat{x}_{j}\right)\right), \quad i=N, \ldots, 1,
$$

where $\operatorname{dec}(\cdot)$ denotes symbol decision that might include channel decoding. When symbol $x_{i}$ is being estimated, it is assumed that the decisions about symbols $\hat{x}_{j}$ with $j=$ $i+1, \ldots, N$ were error-free. Therefore, the signal-to-noise ratio for user $i$ is expressed as

$$
\mathrm{SNR}_{i}=\left|r_{i, i}\right|^{2} \sigma_{x_{i}}^{2} .
$$

Since matrix $\mathbf{Q}$ is unitary, the norm of each column vector $i$ of matrix $\check{\mathbf{H}}$ is equal to its dual column vector $i$ norm from matrix $\mathbf{R}$. Therefore, the previous expression can be rewritten as

$$
\mathrm{SNR}_{i}=\left(\sum_{j=1}^{N}\left|\check{h}_{j, i}\right|^{2}-\sum_{k=1}^{i-1}\left|r_{k, i}\right|^{2}\right) \sigma_{x_{i}}^{2}
$$

The maximum throughput attained by the ZF-based SIC for user $i$ on one given tone is

$$
C_{i}^{\mathrm{ZF}-\mathrm{SIC}}=\log _{2}\left(1+\frac{1}{\Gamma}\left(\sum_{j=1}^{N}\left|\check{h}_{j, i}\right|^{2}-\sum_{k=1}^{i-1}\left|r_{k, i}\right|^{2}\right) \sigma_{x_{i}}^{2}\right) .
$$

\subsection{Iterative MMSE receiver}

The iterative receiver presented here in the context of multiuser DSL transmissions is based on MMSE detection with a priori information. Such a receiver was introduced for turbo-equalization purposes in $[6,12]$ and then adapted for MIMO systems [8]. Information exchanged between the MMSE receiver and the soft-input soft-output (SISO) channel decoder is represented by log-likelihood ratios (LLRs) [13] denoted by $\mathcal{L}(\cdot)$. The LLR on a binary value $b$ is defined by

$$
\mathcal{L}(b)=\ln \left(\frac{P\{b=1\}}{P\{b=0\}}\right) .
$$

As depicted in Figure 2, the MMSE detection provides soft symbols $\widetilde{\mathbf{x}}$ using the channel output $\check{\text { y }}$ and a priori information in the form of soft symbols $\hat{\mathbf{x}}$. During each iteration and for each user, enhanced LLR values $\mathcal{L}_{a}^{d}$ are calculated from the previous ones $\mathcal{L}_{e}^{d}$ by the channeldecoding stage. 


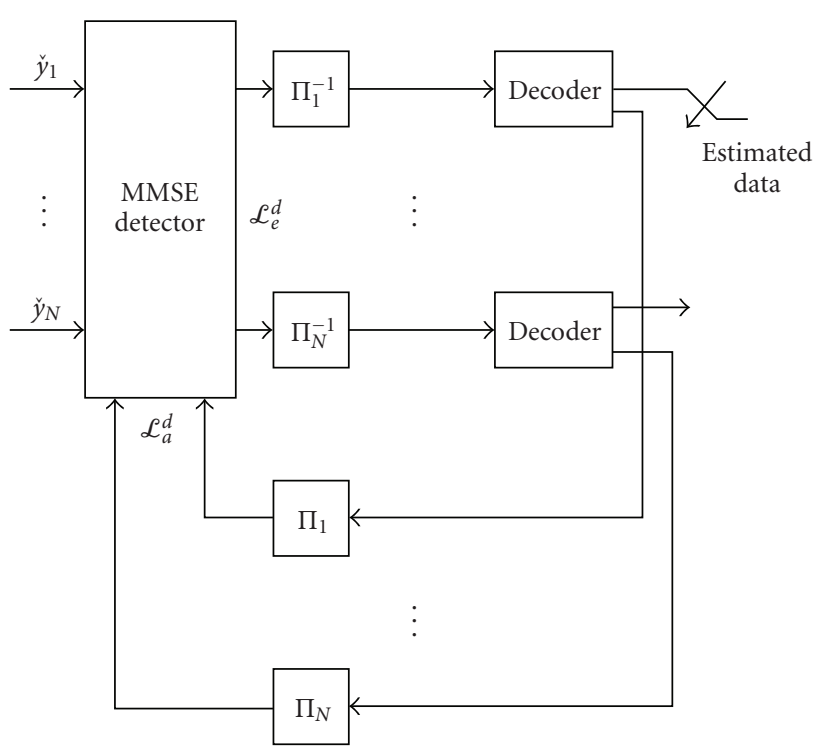

Figure 2: Iterative MIMO receiver.

The decoder requires soft inputs represented by the LLR values of each binary element. It is therefore necessary to convert complex symbols coming from the MMSE detector to LLR values on their binary elements. The output of the MMSE detector can be expressed by

$$
\tilde{x}=g \cdot x+\eta
$$

where $g$ is a bias given by (23) and $\eta$ is noise with a variance of $\sigma_{\eta}^{2}$. The transmitted symbols $x$ are chosen in QAMconstellations $\mathcal{X}$. The conditional probability $P\{\tilde{x} \mid x\}$ is given by

$$
\begin{gathered}
\mathcal{L}\left(b_{i} \mid \tilde{x}\right)=\ln \left(\frac{P\left\{b_{i}=1 \mid \tilde{x}\right\}}{P\left\{b_{i}=0 \mid \tilde{x}\right\}}\right), \\
\mathcal{L}\left(b_{i} \mid \tilde{x}\right)=\ln \left(\frac{\sum_{x \in X_{1}^{i}} P\{x \mid \tilde{x}\}}{\sum_{x \in X_{0}^{i}} P\{x \mid \tilde{x}\}}\right), \\
\mathcal{L}\left(b_{i} \mid \tilde{x}\right)=\ln \left(\frac{\sum_{x \in X_{1}^{i}} P\{\tilde{x} \mid x\} P\{x\}}{\sum_{x \in X_{0}^{i}} P\{\tilde{x} \mid x\} P\{x\}}\right),
\end{gathered}
$$

where $\mathcal{X}_{b}^{i}$ are subsets of constellation $\mathcal{X}$ whose bit of index $i$ is equal to $b$.

In the case of demodulation without a priori information, all symbols are equiprobables. Therefore

$$
\begin{gathered}
\mathcal{L}\left(b_{i} \mid \tilde{x}\right)=\ln \left(\frac{\sum_{x \in X_{1}^{i}} P\{\tilde{x} \mid x\}}{\sum_{x \in X_{0}^{i}} P\{\tilde{x} \mid x\}}\right), \\
\mathcal{L}\left(b_{i} \mid \tilde{x}\right)=\ln \left(\frac{\sum_{x \in X_{1}^{i}} \exp \left(-|\tilde{x}-g \cdot x|^{2} / \sigma_{\eta}^{2}\right)}{\sum_{x \in X_{0}^{i}} \exp \left(-|\tilde{x}-g \cdot x|^{2} / \sigma_{\eta}^{2}\right)}\right) .
\end{gathered}
$$

The decoder delivers LLR values for the decoded binary elements. This information has to be translated to its equivalent complex symbols in order to be fed back to the
MMSE detector. The LLR-to-symbol mapping is performed according to

$$
\begin{gathered}
\hat{x}=E\left\{x \mid \mathcal{L}_{a}^{d}\right\}, \\
\hat{x}=\sum_{s \in \mathcal{X}} s P\left\{x=s \mid \mathcal{L}_{a}^{d}\right\},
\end{gathered}
$$

where $\mathcal{L}_{a}^{d}$ is the a priori LLR value for each binary element contained in the transmitted symbol $x$.

The sets of interleavers $\left\{\Pi_{1} \cdots \Pi_{N}\right\}$ and $\left\{\Pi_{1}^{-1} \cdots \Pi_{N}^{-1}\right\}$ are, respectively, used to arrange the LLR values in the correct order before channel decoding and soft mapping.

The ouput of the MMSE receiver with a priori information can be expressed for each user $i$ of the system by [14]

$$
\tilde{x}_{i}=\lambda_{i} \mathbf{p}_{i}^{H}\left(\check{\mathbf{y}}-\check{\mathbf{H}} \hat{\mathbf{x}}+\hat{x}_{i} \check{\mathbf{h}}_{i}\right),
$$

where $\check{\mathbf{h}}_{i}$ is the $i$ th column of the equivalent channel matrix H. Equation (19) corresponds to interference cancellation followed by MMSE equalization where

$$
\mathbf{p}_{i}=\left(\check{\mathbf{H}}^{\check{H}^{H}}+\mathbf{I}_{N}\right)^{-1} \check{\mathbf{h}}_{i}
$$

is a vector containing the equalization coefficients. It takes into account the reliability of the a priori information by means of the matrix $\mathbf{V}=\operatorname{diag}\left(\hat{v}_{1}^{2}, \ldots, \widehat{v}_{N}^{2}\right)$ with

$$
\hat{v}_{i}^{2}=\sum_{s \in \mathcal{X}_{i}}|s|^{2} P\left\{x=s \mid \mathcal{L}_{a}\right\}-\left|\hat{x}_{i}\right|^{2} .
$$

The coefficients $\lambda_{i}$ are computed as follows:

$$
\lambda_{i}=\frac{\sigma_{x_{i}}^{2}}{1+\left(\sigma_{x_{i}}^{2}-\hat{v}_{i}^{2}\right) \beta_{i}}, \quad \beta_{i}=\mathbf{p}_{i}^{H} \check{\mathbf{h}}_{i} .
$$

The bias $g_{i}$ and the variance $\sigma_{\eta}^{2}$ defined in (15) can be expressed by

$$
\begin{gathered}
g_{i}=\lambda_{i} \beta_{i}, \\
\sigma_{\eta}^{2}=\operatorname{var}\left(\tilde{\mathbf{x}}_{i}-g_{i} \hat{\mathbf{x}}_{i}\right) .
\end{gathered}
$$

At the first iteration in the MMSE receiver, there is no a priori information and $\hat{v}_{i}^{2}=\sigma_{x_{i}}^{2}$. This results in the classical MMSE receiver. In case of perfect a priori information, the interference in the received signal is completely removed and as matrix $\mathbf{V}$ becomes null, the receiver acts as the MRC described in Section 3.2. Then, from (7) and considering a convergence of the iterative process, the maximum throughput of the system with the iterative MMSE receiver on a given tone is expressed as

$$
C_{i}^{\mathrm{MMSE}}=\log _{2}\left(1+\frac{1}{\Gamma} \sum_{j=1}^{N}\left|\check{h}_{j, i}\right|^{2} \sigma_{x_{i}}^{2}\right) .
$$

\section{NUMERICAL RESULTS}

This section is dedicated first to the analysis of the maximum throughput of the different detection schemes presented in Section 4. In a second step, the iterative MMSE receiver behavior is examined in realistic DSL transmission conditions. 


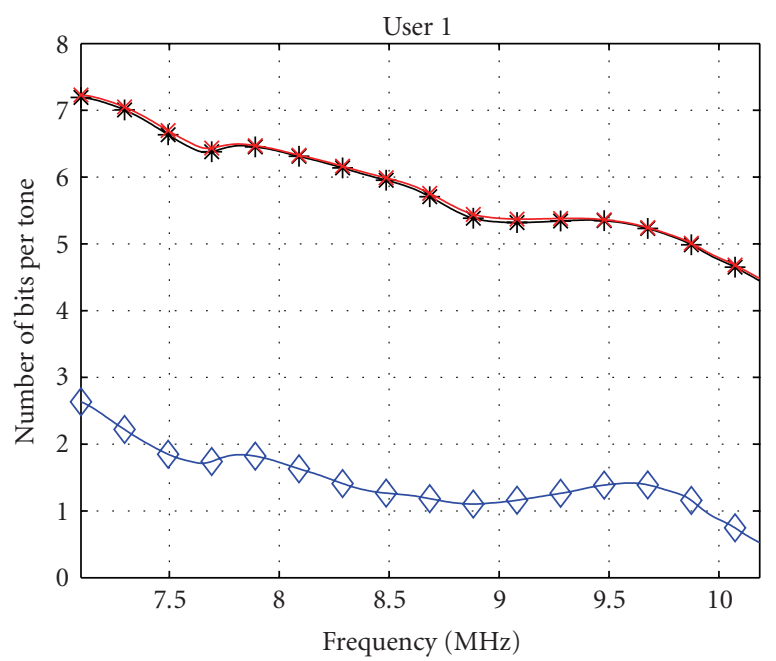

$$
\begin{aligned}
& * \text { Iterative MMSE } \\
& * \text { ZF-SIC } \\
& \succ \text { No FEXT cancellation }
\end{aligned}
$$

(a)

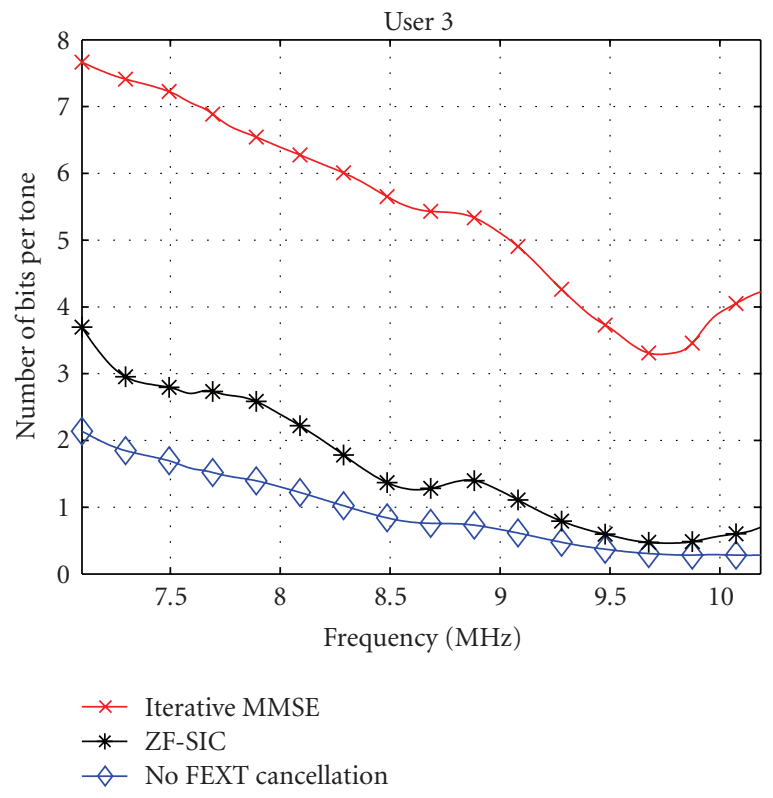

(c)

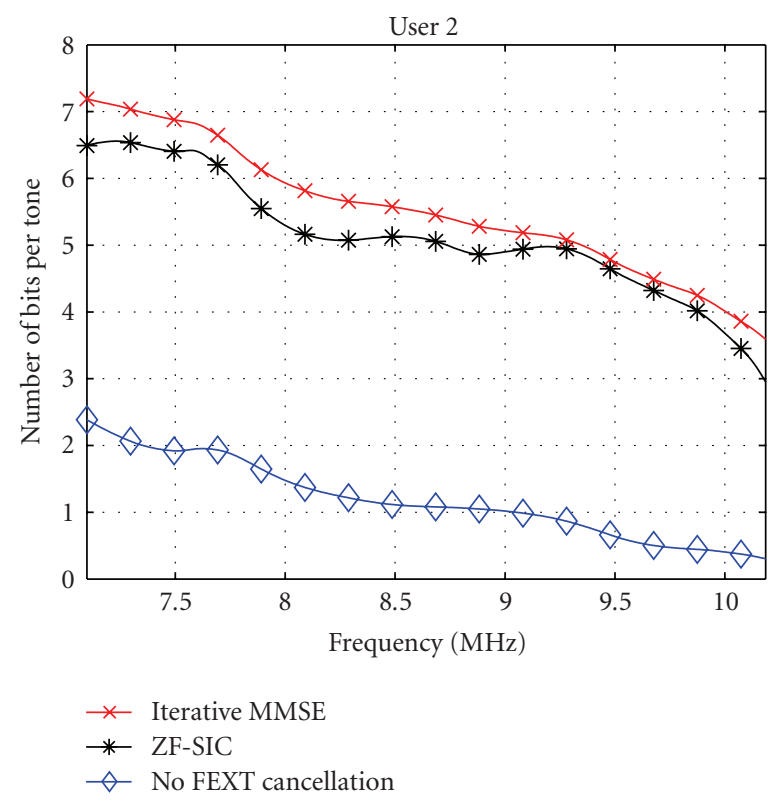

(b)

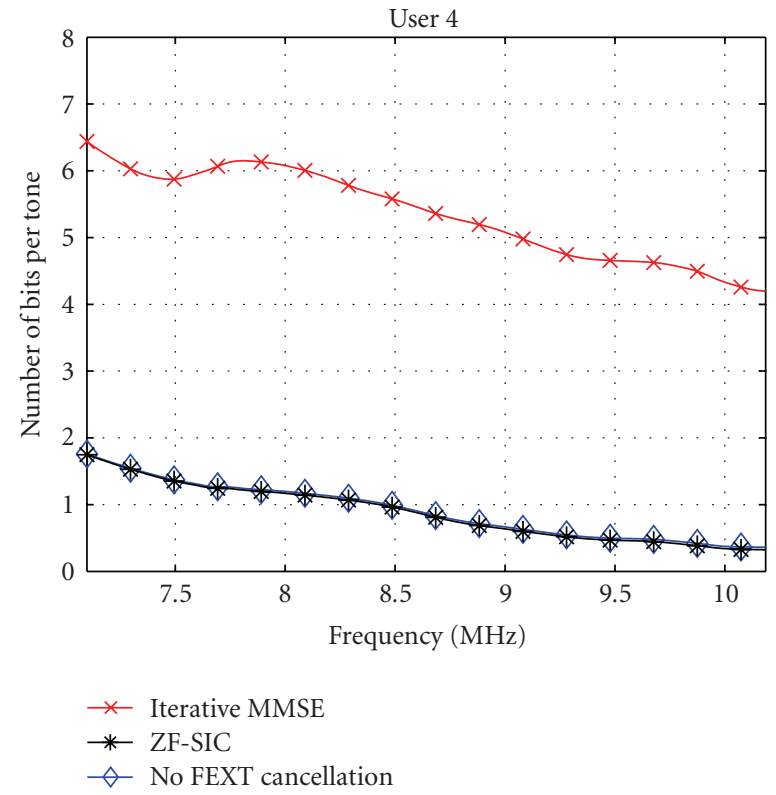

(d)

Figure 3: Iterative MMSE and ZF-SIC bit allocation in the coordinated system.

\subsection{Performance evaluation}

Derived maximum throughput expressions for the ZFbased SIC and iterative MMSE receiver in (13) and (24), respectively, help compare the expected performance of these structures. For this purpose, a scenario of four-coordinated users and two external crosstalk interferers as depicted in Figure 1 is considered. Transmission parameters mimic the VDSL2 setup and are reported in Table 1.

Performance results in terms of allocated bits per tone for each user are drawn in Figure 3. Maximum throughput of the uncoordinated upstream transmission are represented by the diamond curves. The attained maximum throughput by the ZF-SIC scheme is represented by the starred curves and the iterative MMSE performance after convergence is depicted by the crossed curves. The difference in throughput between the coordinated system users is due to the channel structure and the position of the copper pairs in the binder. Moreover, the throughputs decrease with frequency because of the channel attenuation. ZF-SIC performance of user 1 attains the iterative MMSE maximum throughput (24) which has been shown to be equal to the MFB, $C_{1}^{\mathrm{MMSE}}=C_{1}^{\mathrm{ZF}-\mathrm{SIC}}$. Conversely, user 4 does not benefit from the multiuser processing in the ZF-SIC scheme, and its performance is 


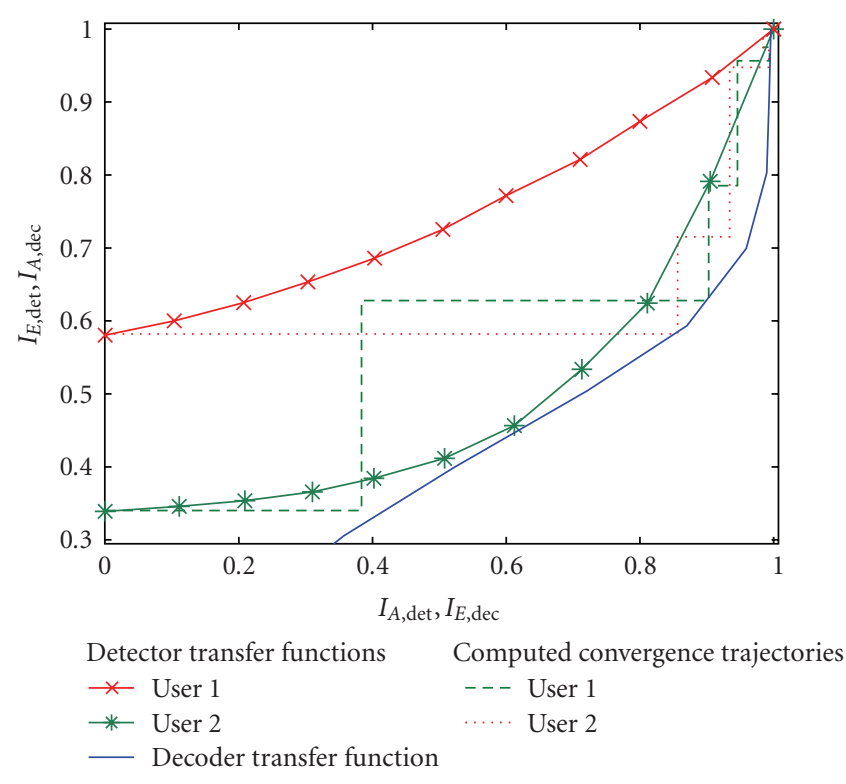

FIGURE 4: EXIT chart for two-coordinated users and one external interferer.

equivalent to single-user transmission performance. On the opposite, all users in the iterative MMSE receiver benefit equally from the multiuser processing to approach the MFB.

\subsection{Iterative MMSE convergence analysis}

The previous results in terms of maximum throughputs assume the convergence of the MMSE iterative processing that reaches the matched filter bound. A practical approach for the iterative MMSE convergence analysis is to use extrinsic information transfer (EXIT) charts introduced by ten Brink in [15]. This allows the exchange of information to be displayed and the transfer characteristics of an iterative process are represented on a chart.

The mutual information between a binary element $x \in$ $\{-1,+1\}$ and its weighted information $\mathscr{L}$ is given by

$$
\begin{aligned}
& I(\mathcal{L}, x) \\
& \quad=\frac{1}{2} \sum_{x \in\{-1,+1\}} \int_{-\infty}^{+\infty} f_{\mathcal{L}}(l \mid x) \log _{2}\left(\frac{2 f_{\mathcal{L}}(l \mid x)}{f_{\mathcal{L}}(l \mid+1)+f_{\mathcal{L}}(l \mid-1)}\right) d l .
\end{aligned}
$$

It is characterized by the probability density function $f_{\mathscr{L}}(\mathcal{L} \mid x)$. This quantity can be approached by a histogram of LLR values (14) which are assumed to follow a Gaussian distribution.

Each block in the iterative MMSE receiver is characterized by a transfer function linking its input and output mutual information [15]. The mutual information transfer function for the detector is plotted with its a priori input $I_{A \text {,det }}$ on the abscissa axis and its extrinsic output $I_{E \text {,det }}$ on the ordinate axis. The decoder component transfer function is plotted with its a priori input $I_{A, \mathrm{dec}}$ on the ordinate axis and its extrinsic output $I_{E, \text { dec }}$ on the abscissa axis. The
TABle 1: System setup.

\begin{tabular}{ll}
\hline Coordinated line length & $600 \mathrm{~m}$ \\
External line length & $75 \mathrm{~m}$ \\
Frequency band & {$[7.09-10.18] \mathrm{MHz}$} \\
Transmitted signal PSD & $\mathcal{E}_{x}=-60 \mathrm{dBm} / \mathrm{Hz}$ \\
External interferer PSD & $\mathcal{E}_{z}=-60 \mathrm{dBm} / \mathrm{Hz}$ \\
White Gaussian noise PSD & $\mathcal{E}_{w}=-140 \mathrm{dBm} / \mathrm{Hz}$ \\
Transmission gap & $9.8 \mathrm{~dB}$ \\
\hline
\end{tabular}

decoder is common to all coordinated users, whereas each user has its own detector transfer function. In the sequel, the convolutional used code is $G(D)=\left(\left(1+D+D^{2}\right),\left(1+D^{2}\right)\right)$ for which a maximum a posteriori MAP decoding algorithm is implemented. Data coding and decoding are carried out in the frequency domain; and each codeword corresponds to a DMT block. Interleaving helps decorrelate data between the detector and the decoder.

Figure 4 shows an EXIT chart for two-coordinated receiving users with one external crosstalk interferer. The mutual information transfer functions are computed using blocks of one hundred DMT symbols. The same parameters setup reported in Table 1 except for the frequency band that is chosen within [7.3-8.9] $\mathrm{MHz}$ is considered. This frequency band results in a bit allocation ranging between 1 and 6 bits per tone.

In an EXIT chart representation, a decoding trajectory will take the form of a stair-shaped curve. The trajectory goes between one of the detector mutual information transfer functions and the decoder transfer function along the different iterations. In the case of two-coordinated lines and one external line, we can prove that the output of the MMSE detector of user 1 depends only on the input of the MMSE detector of user 2 and vice versa, as shown in the appendix. Consequently, the first user trajectory intersects, respectively, the first detector transfer function for the odd iterations and the second detector transfer function for the even iterations. The second user trajectory behaves the opposite way. This progress of the trajectories' iterations is illustrated in Figure 4. The difference between the MMSE detector transfer functions for both users is caused by the structure of the equivalent channel matrix $\check{\mathbf{H}}$. The departing point is low for user 2 because the equivalent channel matrix off-diagonal element $\check{h}_{2,1}$ magnitude is of the same order as the direct path $\breve{h}_{2,2}$ magnitude, whereas the off-diagonal element $\breve{h}_{1,2}$ magnitude is small compared to the direct path $\check{h}_{1,1}$ magnitude.

The same scenario with four-coordinated users and two external crosstalk interferers considered in Section 5.1 is examined regarding convergence of the iterative MMSE process for the setup given in Table 1. The associated EXIT chart is depicted in Figure 5. The resulting EXIT chart and the position of the detector mutual information transfer 


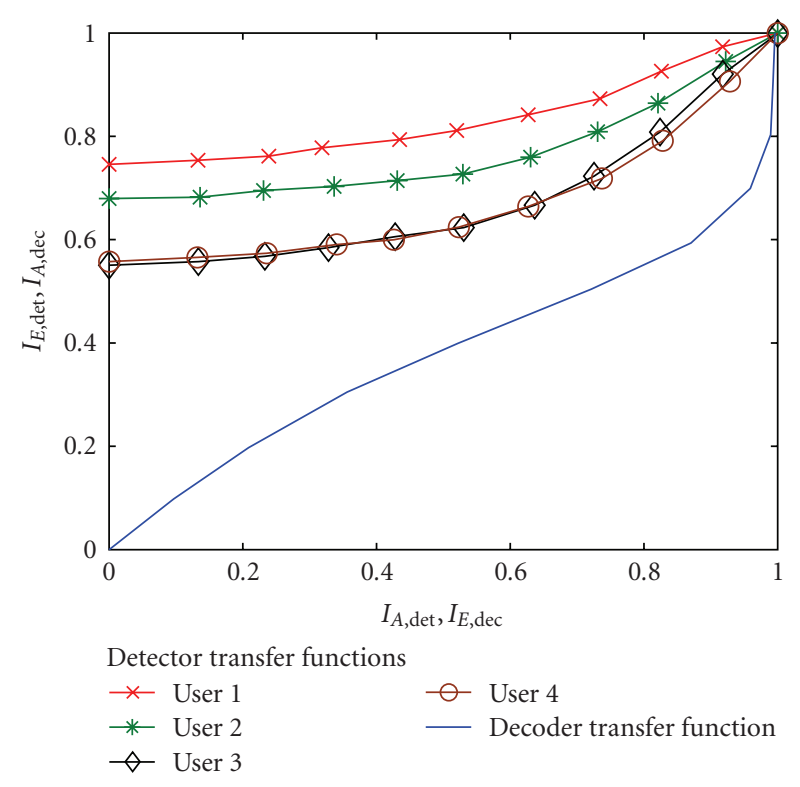

FIGURE 5: Convergence of the iterative system for four-coordinated users with two external crosstalk interferers.

functions indicate that the system converges within few iterations and therefore the matched filter bound performance will be attained.

\section{CONCLUSION}

In this paper, the combination of external crosstalk whitening and iterative MMSE processing is examined for upstream coordinated DSL systems. The iterative MMSE receiver achieves the MFB performance under the assumption of perfect a priori information. Unlike the ZF-SIC, all users in the iterative MMSE receiver benefit equally from the multiuser processing to approach the MFB. EXIT charts are used to analyze the behavior of this scheme for upstream coordinated DSL systems in the presence of correlated external noise. Convergence of the iterative MMSE receiver for coordinated DSL systems is obtained in realistic conditions.

\section{APPENDIX}

Lemma A.1. In the MIMO system with an iterative MMSE detector for two-coordinated users and without a priori information fed to the soft demapper, the output information for user 1 depends only on the input information for user 2.

Proof. The output signal for user 1 with interference cancellation can be written as

$$
\begin{gathered}
\tilde{x}_{1}=\lambda_{1} \mathbf{p}_{1}^{H}\left(\mathbf{y}-\check{\mathbf{H}} \hat{\mathbf{x}}+\hat{x}_{1} \check{\mathbf{h}}_{1}\right), \\
\tilde{x}_{1}=\lambda_{1} \mathbf{p}_{1}^{H}\left(\mathbf{y}+\widehat{x}_{2} \check{\mathbf{h}}_{2}\right) .
\end{gathered}
$$

Developing the matrix computation of $\lambda_{1} \mathbf{p}_{1}^{H}$, we show that

$$
\begin{aligned}
\lambda_{1} \mathbf{p}_{1}^{H}= & \frac{\sigma_{x}^{2}}{A v_{2}^{2} \sigma_{x}^{2}+B \sigma_{n}^{2} \sigma_{x}^{2}+C v_{2}^{2} \sigma_{n}^{2}+\sigma_{n}^{4}} \\
& \times\left[\begin{array}{c}
\left(\check{h}_{11}^{*}\left|\check{h}_{22}\right|^{2}-\check{h}_{21}^{*} \check{h}_{12}^{*} \check{h}_{22}^{*}\right) v_{2}^{2}+\check{h}_{11}^{*} \sigma_{n}^{2} \\
\left(\check{h}_{21}^{*}\left|\check{h}_{12}\right|^{2}-\check{h}_{11}^{*} \check{h}_{22}^{*} \check{h}_{12}^{*}\right) v_{2}^{2}+\check{h}_{21}^{*} \sigma_{n}^{2}
\end{array}\right]^{T}
\end{aligned}
$$

with

$$
\begin{aligned}
A= & \left|\check{h}_{11}\right|^{2}\left|\check{h}_{22}\right|^{2}+\left|\check{h}_{21}\right|^{2}\left|\check{h}_{12}\right|^{2} \\
& -\check{h}_{11} \check{h}_{21}^{*} \check{h}_{12}^{*} \check{h}_{22}-\check{h}_{21} \check{h}_{11}^{*} \check{h}_{22}^{*} \check{h}_{12}, \\
B= & \left|\check{h}_{11}\right|^{2}+\left|\check{h}_{21}\right|^{2}, \\
C= & \left|\check{h}_{22}\right|^{2}+\left|\check{h}_{12}\right|^{2} .
\end{aligned}
$$

Therefore, $\tilde{x}_{1}$ depends only on the reliability of user 2 .

\section{REFERENCES}

[1] G. Ginis and C.-N. Peng, "Alien crosstalk cancellation for multipair digital subscriber line systems," EURASIP Journal on Applied Signal Processing, vol. 2006, Article ID 16828, 12 pages, 2006.

[2] G. Ginis and J. M. Cioffi, "Vectored transmission for digital subscriber line systems," IEEE Journal on Selected Areas in Communications, vol. 20, no. 5, pp. 1085-1104, 2002.

[3] R. Cendrillon, G. Ginis, E. van den Bogaert, and M. Moonen, "A near-optimal linear crosstalk canceler for upstream VDSL," IEEE Transactions on Signal Processing, vol. 54, no. 8, pp. 31363146, 2006.

[4] V. Le Nir, M. Moonen, and J. Verlinden, "Optimal power allocation under per-modem total power and spectral mask constraints in xDSL vector channels with alien crosstalk," in Proceedings of the IEEE International Conference on Acoustics, Speech and Signal Processing (ICASSP'07), vol. 3, pp. 357-360, Honolulu, Hawaii, USA, April 2007.

[5] X. Wang and H. V. Poor, "Iterative (turbo) soft interference cancellation and decoding for coded CDMA," IEEE Transactions on Communications, vol. 47, no. 7, pp. 1046-1061, 1999.

[6] M. Tüchler, A. C. Singer, and R. Koetter, "Minimum mean squared error equalization using a priori information," IEEE Transactions on Signal Processing, vol. 50, no. 3, pp. 673-683, 2002.

[7] J. Le Masson, C. Langlais, and C. Berrou, "Linear precoding with low complexity MMSE turbo-equalization and application to the wireless LAN system," in Proceedings of the IEEE International Conference on Communications (ICC '05), vol. 4, pp. 2352-2356, Seoul, Korea, May 2005.

[8] M. Witzke, S. Bäro, F. Schreckenbach, and J. Hagenauer, "Iterative detection of MIMO signals with linear detectors," in Proceedings of the 36th Asilomar Conference on Signals, Systems and Computers (ACSSC '02), vol. 1, pp. 289-293, Pacific Grove, Calif, USA, November 2002.

[9] Y. C. Eldar and A. V. Oppenheim, "MMSE whitening and subspace whitening," IEEE Transactions on Information Theory, vol. 49, no. 7, pp. 1846-1851, 2003. 
[10] S. Venkatesan, L. Mailaender, and J. Salz, "An iterative algorithm for computing a spatial whitening filter," in Proceedings of the 5th IEEE Workshop on Signal Processing Advances in Wireless Communications (SPAWC '04), pp. 338-342, Lisbon, Portugal, July 2004.

[11] J. G. Proakis, Digital Communications, McGraw-Hill, New York, NY, USA, 1995.

[12] A. Glavieux, C. Laot, and J. Labat, "Turbo equalization over a frequency selective channel," in Proceedings of the International Symposium on Turbo Codes and Related Topics (ISTC '97), pp. 96-102, Brest, France, September 1997.

[13] J. Hagenauer, E. Offer, and L. Papke, "Iterative decoding of binary block and convolutional codes," IEEE Transactions on Information Theory, vol. 42, no. 2, pp. 429-445, 1996.

[14] C. Laot, R. Le Bidan, and D. Leroux, "Low-complexity MMSE turbo equalization: a possible solution for EDGE," IEEE Transactions on Wireless Communications, vol. 4, no. 3, pp. 965-974, 2005.

[15] S. ten Brink, "Convergence behavior of iteratively decoded parallel concatenated codes," IEEE Transactions on Communications, vol. 49, no. 10, pp. 1727-1737, 2001. 

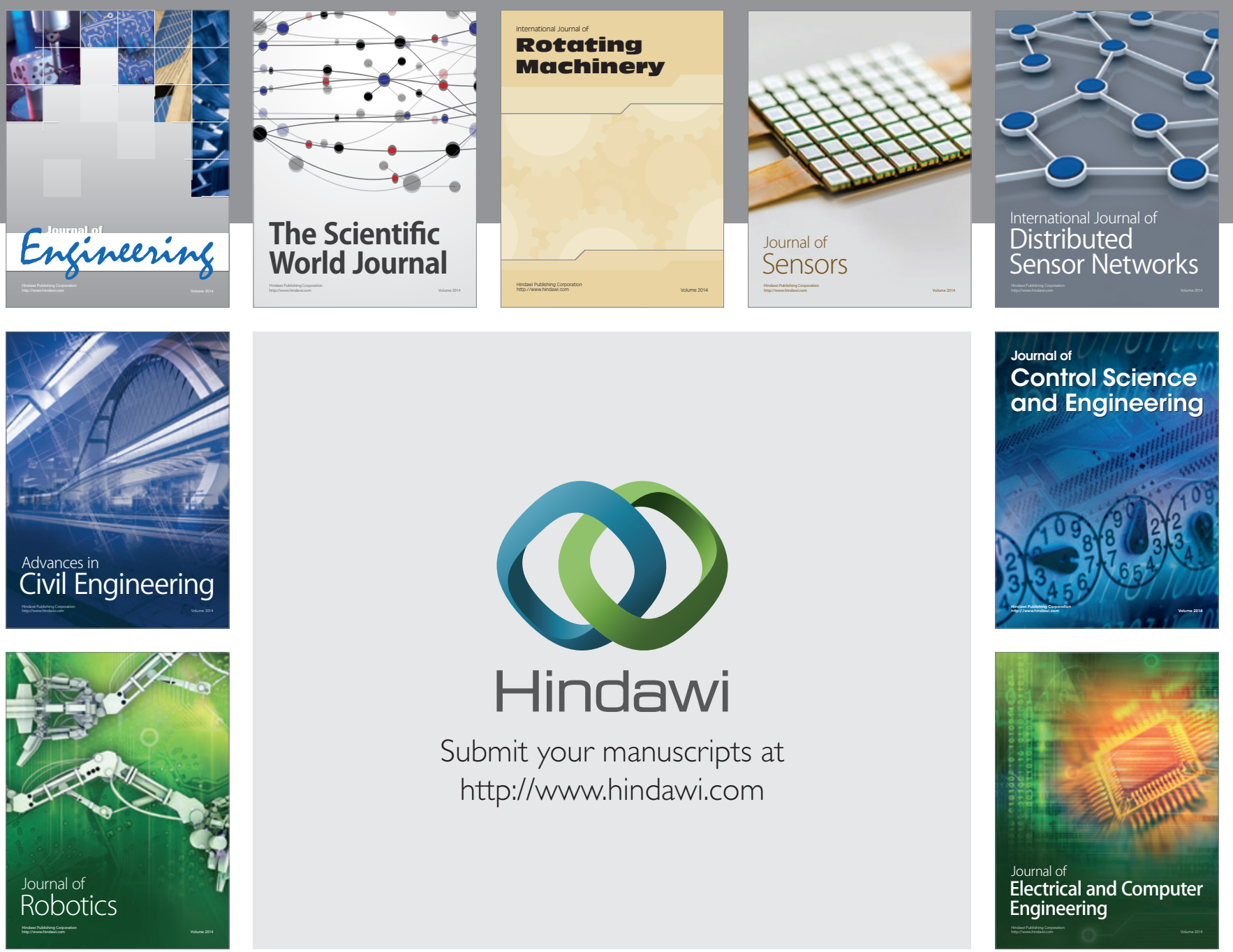

Submit your manuscripts at

http://www.hindawi.com
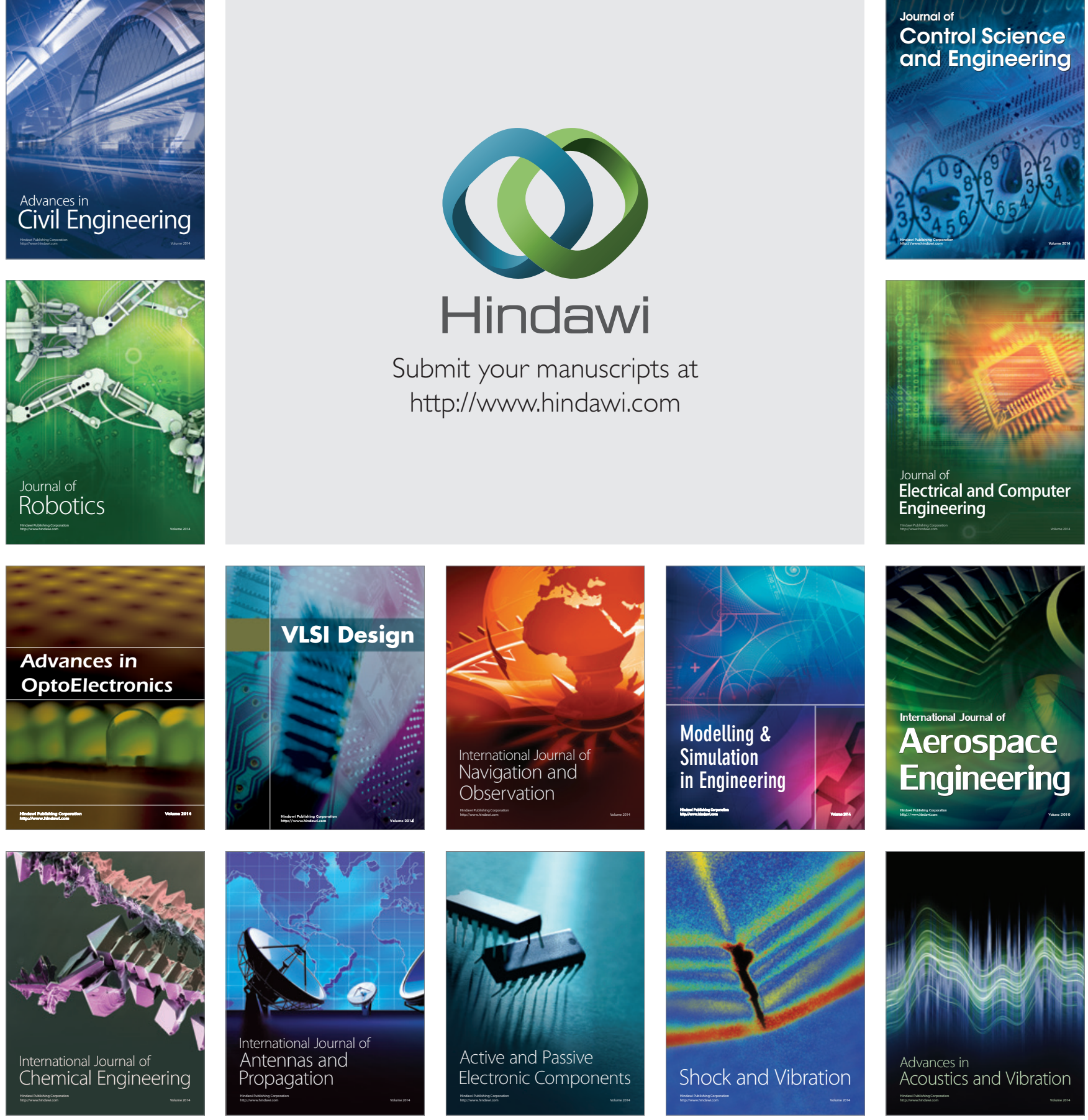